\title{
Identification of high energy gamma-ray sources and source populations in the era of deep all-sky coverage
}

Received: date / Accepted: date

\begin{abstract}
A large fraction of the anticipated source detections by the Gamma-ray Large Area Space Telescope (GLAST-LAT) will initially be unidentified. We argue that traditional approaches to identify individuals and/or populations of gamma ray sources will encounter procedural limitations. Those limitations are discussed on the background of source identifications from EGRET observations. Generally, our ability to classify (faint) source populations in the anticipated GLAST dataset with the required degree of statistical confidence will be hampered by sheer source wealth. A new paradigm for achieving the classification of gamma ray source populations is discussed.
\end{abstract}

Keywords gamma rays · observations - methods: data analysis

PACS 95.85.Pw $\cdot 98.70 . \mathrm{Rz}_{\mathrm{z}} \cdot 95.75 . \mathrm{-z}$

\section{Problem statement}

The anticipated source wealth from observations carried out by the satellite-based $\gamma$-ray mission GLAST, potentially yielding the discovery of thousands of new highenergy sources following extrapolations from predecessor experiments, will create several problems for source identification. Catalogs of the most prominent candidate

\footnotetext{
O. Reimer

W.W. Hansen Experimental Physics Laboratory \& Kavli Institute for Particle Astrophysics and Cosmology Stanford University

Stanford, CA 94305-4085, USA

Tel.: +1-650-724-6819, Fax: +1-650-725-2463

E-mail: olr@stanford.edu

D. Torres

Institució de Recerca i Estudis Avançats (ICREA) \& Institut de Ciències de l'Espai (IEEC-CSIC)

Facultat de Ciencies, Universitat Autònoma de Barcelona

Torre C5 Parell, 2a planta

08193 Barcelona, Spain

Tel.: +34-93-581-4352 Fax: +34-93-581-4363

E-mail: dtorres@ieec.uab.es
}

sources (Active Galactic Nuclei -AGNs-, and neutron stars/pulsars -PSRs-) will very likely not be complete to the required low radio and/or X-ray flux levels required for counterpart studies (AGN), or do not have the ability to provide a suitable counterpart at all (radioquiet PSRs). Predictably, this will leave many of the new $\gamma$-ray source detections initially unidentified. And even if the pulsar and AGN catalogs were sufficiently deep, they may not yield unambiguous source identifications: A complete catalog for the anticipated numbers of sources, projected using the instrumental point-spreadfunction (psf), would generate total sky coverage, with one or more candidates in every line-of-sight for incident photons corresponding to their (energy dependent) psf. There would be one or more AGN everywhere, and one or more pulsar in every line-of-sight at low galactic latitude. This will limit or even prevent unambiguous source identifications based solely on spatial correlation. In addition, a legacy from the EGRET experiment is the indication that we are already missing the finite identification of one or more source populations, both at low and at high Galactic latitude. Specifically, the identification of variable, non-periodic, point-like sources at low galactic latitude, as well as of non-variable sources at high latitude is still missing [1], since these source populations exhibit characteristics different from the EGRETdetected pulsars or blazars.

In the GLAST era and beyond, if it is the objective to conclusively identify all individual $\gamma$-ray source detections, we will predictably fail. The anticipated number of counterparts, their relative faintness deduced from luminosity functions, the missing all-sky coverage in the relevant wavebands for deep counterpart studies, and the expected ambiguities due to source confusion in densely populated regions of the $\gamma$-ray sky will preclude reaching this ultimate goal of source identification. Consequently, we should aim to identify at least all classes of sources, and subsequently attempt to gain in-depth astrophysical knowledge by studying the most interesting or prominent representatives among such populations. The anticipated number of source detections left unidentified will pre-

Contributed to The Multi-Messenger Approach to Unidentified Gamma-Ray Sources: 3rd Workshop

on the Nature of Unidentified High-Energy Sources, 4-7 July 2006, Barcelona, Spain

Submitted to Astrophys.Space Sci.

Work supported in part by US Department of Energy contract DE-AC02-76SF00515 


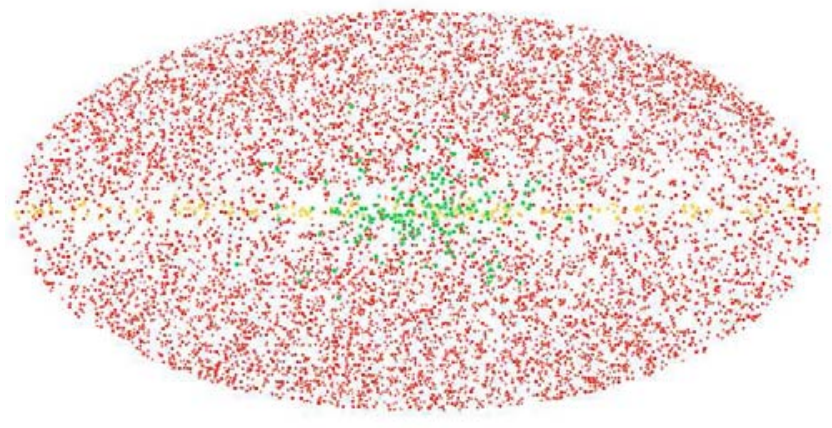

Fig. 1 Synthetic gamma-ray source catalog for GLAST-LAT observations, based on a flux-limited source sample according to a realistic diffuse gamma-ray emission model. AGN (red dots) dominate the catalog. Two additional source populations have been considered: Sources in the Galactic bulge (yellow), and a Galactic halo source population (green). Figure credits: Seth Digel

clude individual deep multifrequency studies for every source, in the way it led to the identification of e.g. the Geminga pulsar and various $\gamma$-ray blazars.

Suppose that we have a sufficiently complete counterpart catalog, such that a member of it spatially coincides with most of the GLAST-LAT sources. Does this imply that we have already identified all sources? To answer this question consider that we have, instead, a reasonably complete sky coverage of sources, i.e. GRBs as an example. An overlay of all error boxes of GRBs reported from BATSE covers the whole sky. Then, there is at least one GRB spatially coinciding with any possible counterpart or host. Consequently, here a spatial correlation analysis lacks identification capability, even when it is clear that not all populations of astrophysical objects are plausible candidates for GRB generation or hosting, nor that all of them should even be probed. More particularly, we can not claim, using correlation analysis, that GRBs have appeared more often in starburst or luminous infrared galaxies than in normal galaxies. Therein lies the dilemma. If the number of unidentified sources and/or the number of plausible candidates is sufficiently large, what will constitute a sound identification? How shall we find evidence for new populations of sources, and new members within these populations, in the GLAST era?

At present, the most successful identification scheme for $\gamma$-ray sources is based upon multifrequency followup observations, unless there is a given prediction of periodicity, which itself would unambiguously label the source if the same periodicity is found in the $\gamma$-ray data. The latter, however, will happen only for a fraction of GLAST-LAT detections, either because of the absence of contemporaneous pulsar timing solutions (in particular for X-ray pulsars), or sufficient statistical significance for claiming periodicity in the still photon-limited $\gamma$-ray data, or because of shortage of precise theoretical predictions for testable variability patterns other

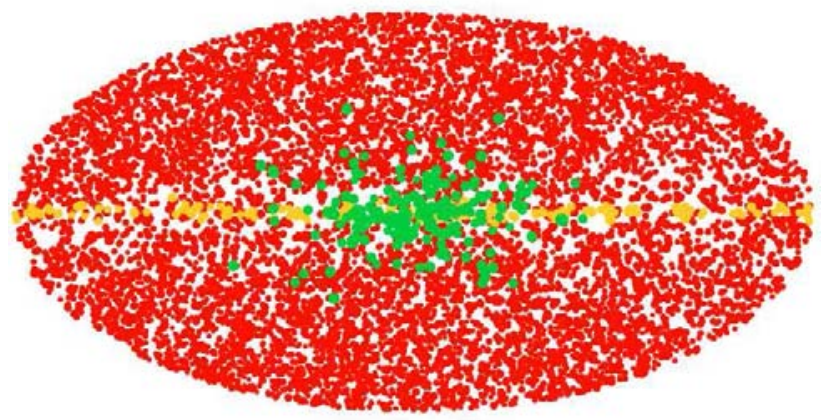

Fig. 2 Synthetic gamma-ray source catalog as in Fig.1, but symbol size has been enlarged to represent source location uncertainty contours as expected for a large source catalog: At almost any line of sight there is a gamma-ray source found in their respective error circle. This will indicate the problem of probing the existence of new source populations in the GLAST-LAT era. Log N-log S predictions for the increased instrumental sensitivity predict vastly more sources than we know of today (EGRET), and the majority of them are supposedly faint sources below the EGRET detection limit, thus not tremendously better localized by GLAST-LAT.

than periodicity. Note that variability of $\gamma$-rays probes, generally, timescales, not periodicities, and can be used predominantly to rule out membership into classes and only when it could be established at a significant level. For example, if a given source is variable, we consequently assume that it is not produced in phenomena on timescales larger than the corresponding exposure. In essence, this will rule out all possible counterparts producing steady $\gamma$-ray fluxes. In fact, for many of the theoretically anticipated LAT sources steady $\gamma$-ray emission is predicted. Such candidate populations are Supernova remnants (e.g., [2]), luminous infrared galaxies (e.g., [3]), or galaxy clusters (e.g., 44).

However, if a theoretically compatible variability timescale exists, it will prompt the need of carrying out followup observations, which will necessarily require a considerable amount of time and resources, without guaranteed success of achieving an unique identification. The bottom line is that adopting this scheme, with GLAST observations, particularly during the first year of data taking, we may limit our capability to identify new populations of sources if relying exclusively on multifrequency follow-up methods for source identification.

If we have a spatial coincidence between a candidate and an unidentified $\gamma$-ray source, and in addition there exist a matching variability timescale between theoretical predictions for such object and the data, then how can we, with nothing else, definitely say that an identification was achieved? And even if we convince ourselves to assert it, how many of such individual cases should be found in order to claim the discovery of a new population of sources with satisfactory statistical significance? How would the latter be quantitatively evaluated? Not having a priori of the expected number of source detections a 
criterion by which to answer the previous questions will confront us with a situation of ambiguity between results achieved by applying different classification standards, with no instance to decide in a unbiased way whether an identification has been achieved or not.

In order to overcome these predictable problems, a paradigm shift in the way we seek the classification of $\gamma$-ray populations is suggested. We need to define a sensitive and quantitative criterion, by which we could identify both variable and non-variable populations. A feasible scheme for defining such a criterion was laid out in [5], and is referred to in the following. Although we refer here explicitly to the case of GLAST-LAT, more particular $\gamma$-ray source detection with the Large Area Telescope (LAT), the scheme we present is adaptable to other experiments confronted with a similar combination of problems, for example in hemispheric neutrino astronomy.

\section{Identification of $\gamma$-ray populations}

Here we elaborate a scheme to identify and classify new $\gamma$-ray source populations.

\subsection{What to search for?}

Starting from a given theoretical prediction of a population of astronomical objects to be detectable above the LAT instrumental sensitivity, we propose to impose a

- Theoretical censorship: we request as part of the criterion that predictions, ideally of multiwavelength character, are available for a subset of the proposed class of counterparts. The term predictions refers here to measurable observables for the respective instrument.

This request is made to avoid the blind testing of populations that may or may not produce $\gamma$-rays, but for which no other than a spatial correlation result can be achieved a posteriori. If there is no convincing theoretical support that a population can emit $\gamma$-rays before conducting the search, such population may not be sought this way. Although obvious, it should be explicitly stated that we will not, by applying this method, disallow the possibility of making serendipity discoveries. Imposing of a theoretical censorship is not just a matter of theoretical purity, but rather it is statistically motivated, as we explain below. Such censorship applies similarly to all a priori selection of subclasses, i.e., the imposing of cuts in samples that are aimed to isolate the members from which we preferably expect detectable $\gamma$-ray emission.

\subsection{Protection of discovery potential}

By probing a large number of counterparts candidates with at least equally large number of trials with the same data set, one will find positive correlations, at least as a result of statistical fluctuations (also referred to as chance capitalization). Then, to claim significance, one would have to check if the penalties that must be paid for such a finding (i.e., the fact that there were a number of trials that led to null results) does not overcome the significance achieved. Needless to say, a number of possible bias are expected to influence the computation of the penalties. The example here is ultra high energy cosmic rays (UHECRs), where there are already a number of dubious discovery claims from correlation studies, even when the sample of events is small (see, e.g. [6], 7]). GLAST-LAT, and in general $\gamma$-ray astronomy, can prepare to address this difficulty before entering the new era of source wealth, as UHECR physics does before unblinding data from the Pierre Auger observatory ([8]). In this sense, this part of our criterion is rather similarly defined. We require an

- A priori protocol: The populations that are to be tested in the GLAST-LAT data shall be defined before the initial data release.

A protocol is technically a budget for testing correlations. Every test will consume part of this budget up to a point that, if we still proceed in testing, there can be no statistical significant detection claim achieved anymore. A protocol secures that a detection of a population can be made with confidence in its statistical significance for a number of interesting classes. As remarked by the 8 , when confronted with claims made in the absence of an a priori protocol, one may assume that a very large number of failed trials were made in order to find the positive results being reported, and thus disregard the claims altogether just by denying statistical weight. Otherwise stated, we might be asked for proof that the penalty for failed trials has been accounted for and is indeed below a required statistical significance. This may turn out to be, either very difficult to achieve or strictly impossible because of the possible biases in penalties definitions.

Additional exploration of the same data set for expected or unexpected populations can (and certainly will) be made, although if the budget is spent, without the strength of immediate discovery potential. A positive additional search must be thought of as a way of pointing towards new populations of sources to be tested with additional or independent sets of data then. Here, a source catalog based on the second year of GLAST-LAT observations would not be independent: it will combine already discovered persistent sources with newly discovered ones that were below the instrumental sensitivity or imposed detection threshold beforehand, or of transient character.

Summarizing, if using the same set of data, claiming the discovery of one population affects the level of confidence by which one can claim the discovery of a second. Then, suppose for definiteness that the total budget is a chance probability equal to $\mathcal{B}$, e.g., $10^{-4}$. That is, 
that a claim for population(s) discovery has to be better than one having a probability of chance occurrence equal to $\mathcal{B}$, and that we want to test $A, B, C \ldots$ classes of different sources (say, radio galaxies, starburts galaxies, microquasars, pulsars, AGN, for a recent overview see [9). The total budget can then be divided into individuals, a priori, chance probabilities, $P_{A}, P_{B}$, etc., such that $\sum_{i} P_{i}=\mathcal{B}$. This implies that population $i$ will be claimed as detected in this framework if the a posteriori, factual, probability for its random correlation, $P^{\mathrm{LAT}}(i)$, is less than the a priori assigned $P_{i}$ (as opposed to be less only than the larger, total budget). The $\sum_{i} P_{i}$ also accounts for any attempt to investigate population properties of subsamples belonging to the same object class by invoking cuts. If too many subsamples were investigated in order to discriminate further among the emission characteristics in an already detected source population, such selections are on the expense of the budget, too. Statistically dependent test shall be avoided. A minimal set of subsamples, imposing substantially different cuts in their selections, is the most adequate choice to maximize the chance for statistically-significant classifications of subsamples.

We could go a step forward and suggest to manage the budget of probabilities. For some populations, e.g., those which were not detected in EGRET observations, we can less confidently assume that they will be detected, or perhaps for some others, the number of their members may be low enough such that a detection of only several of its individuals would be needed to claim a large significance. In this situation we would choose a relatively higher $P_{i}$, so that it would be easier to find $P^{\mathrm{LAT}}(i)<P_{i}$. For others, say AGN and pulsars, we are confident that they will be detected, and thus we would be less willing to spent a large fraction of the discovery budget in them. Within the protocol, we can statistically prove that these population appear with very high confidence by assigning a very low $P_{i}$ in such a way to make harder for the test to pass. If one or more of the tests, i.e., if for several $i$-classes, $P^{\mathrm{LAT}}(i)<P_{i}$, is fulfilled, the results are individually significant. First, because we protected our search by the a priori establishment of the protocol (a blind test) and second, because the overall chance probability is still less than the total budget $\mathcal{B}$.

We refrain ourselves here to explicitly propose which are the populations to be tested and how large the a priori probability assigned to each of them as well as the exact number for the total budget $\mathcal{B}$ should be. This ultimately has to be carefully studied by the GLASTLAT collaboration for data in the proprietary period, although obvious choices can be compiled and argued. Now we proceed towards a most delicate issue, that of the treatment of the statistical significance of claimed detections of source populations.

\subsection{How to search and significance assessment}

The last constituent of a methodological approach to identify new classes of $\gamma$-ray sources is the application of a

- Common significance assessment: we urge that a strict statistical evaluation is mandatory before a claim of a discovery of a new source population can be made. An objective method is presented in the following.

We start by assessing the number of members of the relevant candidate class being probed, for which predictions exist, that coincide with GLAST-LAT source detections of unidentified $\gamma$-ray sources. Let $\mathcal{C}(A)$ represent this number for population $A$. In what follows, for the sake of simplicity, we will assume that we deal with equally probable coincidences, when a projected position is less distant than, say, the $95 \%$ confidence contour.

Let $\mathcal{N}(A)$ be the number of known sources in the particular candidate population $A$ under analysis and $\mathcal{U}$ the number of LAT detections. Let $\mathcal{P}$ be the probability that in a random direction of the sky we find a LAT source. The probability $\mathcal{P}$ should take into account instrumental detectability issues (exposure gradients, imprecision of the diffuse emission model, etc.) as well as, at low Galactic latitudes, expected Galactic structures.

As an example which omits the latter complications, one may use angular coverage (the ratio between the area covered by $\mathcal{U}$ sources and that of the sky region upon which these sources are projected). In what follows, we will assume that such method is in place for LAT and that $\mathcal{P}$ can be computed for a given region of the sky. Note that to compute $\mathcal{P}$ we do not need any information about the candidates, but just some sensible extrapolation of the expected number of detections of sources that have been already identified. The value of $\mathcal{P}$ is obtained a priori of checking for any population.

Whatever the method, $\mathcal{P}$ is expected to be small for LAT. To give an example, if we take just a coverage assessment at high Galactic latitudes $(|b|>10)$, and we assume that there will be a thousand detections, and that the typical size of the error box of LAT sources is a circle of radius $12 \operatorname{arcmin}$, then $\mathcal{P} \sim 3 \times 10^{-3}$. At lower latitudes, we expect $\mathcal{P}$ to be between 1 to 2 orders or magnitude larger. We believe that a more careful treatment of source number predictions and the range of expected source location uncertainties will reduce the value of $\mathcal{P}$ from such simple estimations. Such low values for $\mathcal{P}$ make the product $\mathcal{P} \times \mathcal{N}(A)$ typical less than $1-10$, for all different candidate populations. We will refer to this product as the noise expectation, i.e., this is the number of coincidences which one would expect even when there is no physical connection between the LAT detections and population $A$. 
The number of excess detections above noise will be, $\mathcal{E}(A)=\mathcal{C}(A)-\mathcal{P} \times \mathcal{N}(A) \mathbb{1}$ Two cases can be distinguished. The two largest populations of plausible candidates (pulsars and blazars) will also present the largest number of coincidences, since it is already proven that they do emit high energy $\gamma$-rays above LAT sensitivity, and the populations are sufficiently large in number. Let's assume that there are 2000 catalogued AGNs; with the quoted value of $\mathcal{P}$, all coincidences in excess than 6 are beyond the random expectation. The reality of the population in the EGRET catalog make us expect that $\mathcal{C}(\mathrm{AGN}) \gg 6$, and thus that the number of excesses would be equally large. In this case, we are in the domain of large number statistics and a probability for the number of excesses to occur by chance, $P^{\mathrm{LAT}}(\mathrm{AGN})$ can be readily computed.

A different case appears when the second term in the expression for $\mathcal{E}(A)$ is a small quantity. Two scenarios may be found: if the number of coincidences for that population is large compared with the noise, we are again in the domain of large number statistics, as in the case of AGN or pulsars. This will -most likely- not happen for many (or perhaps for any) of the new populations we would like to test. Thus, in general we are in the realm of small number statistics: we should test the null hypothesis for a new source population against a reduced random noise (see [10, also [11]) 2

Let us analyze now an explicit example. We are testing a null hypothesis (e.g., X-ray binaries are not LAT sources). That is represented by 0 predicted signal events (coincidences), i.e. total number of events equal to the background in Table 2-9 (see leftmost columns) of 10 . Suppose for definiteness that $\mathcal{P} \sim 3 \times 10^{-3}$ and $\mathcal{N}(A)$ is equal to, say, 200, then the number of chance coincidences (the noise or background) is 0.5 . Thus, if we find more than 5 individual members of this class (e.g. superseding the confidence interval 0.00-4.64) correlated with

1 Obviously, if the number of sources is so large that $\mathcal{P} \rightarrow 1$, then $\mathcal{E}=0$. If instead, the number of members in the potential counterpart class is so large that $\mathcal{C}(A) \rightarrow \mathcal{P N}(A)$, then $\mathcal{E}=0$ too. In both cases, there is no way to distinguish whether the population is physically associated. To simplify the treatment we consider excesses with no overlapping, i.e., coincidences between members of population A and LAT sources that are not co-spatial with members of other populations. In reality, the available $\gamma$-ray observables will allow further discrimination, either directly by reducing overlap between members of different populations at higher photon energies (better source localization due to narrower instrumental psf), or when the populations under consideration become distinguishable due to their source spectra, and variability pattern.

2 If a precise number of detectable sources is predicted, generally one could test the hypothesis of their presence in the LAT catalog directly, using small number statistics described in more detail below. However, this will unlikely constitute the standard scenario since we will not know precisely from theoretical arguments how many, say, of the X-ray binaries, should indeed be detectable. Modeling is in general not applied with an equal level of detail to a sufficiently high number of members in a candidate population.
LAT sources, we have proven that the null hypothesis is ruled out at the the $95 \%$ CL.

Using the small number statistics formalism, we can convert the level of confidence achieved for each population into the factual probability, i.e., $P^{\mathrm{LAT}}$ (X - ray bin). Subsequently, by compfaring with the a priori budgeted requirement (i.e., is $P^{\mathrm{LAT}}\left(\mathrm{X}-\right.$ ray bin.) $<P_{\mathrm{X}-\text { ray bin.? }}$, we will be able to tell whether the population has been discovered. Clearly, if instead we find no more than 5 individual sources in the same example, then we have no evidence by which to claim the existence of this population at that level of confidence.

Managing $P_{A}$ is equivalent to requesting different populations to appear with different, intelligently selected, levels of confidence. By using this method, detecting just a few members of each class may allow to achieve significant levels of confidence, justified by the existence of the imposed theoretical censorship and protected by an a priori protocol. Note that at this stage there is no variability analysis involved. If we were to add the search on compatible variability timescales, the confidence level of the detections will even improve.

\section{Concluding remarks}

The proposed criterion for identification of $\gamma$-ray source populations integrates three different parts: 1) A theoretical censorship that prohibits executing repeated searches that would reduce the statistical significance of any possible positive class correlation. 2) An a priori protocol that protects the significance by which to claim the discovery of a number of important population candidates and gives guidelines as to how to manage the probability budget 3) A significance assessment that assigns probabilities both in the large and in the small numbers statistical regime.

It is useful to note that LAT will be in a privileged position to actually identify new population of sources. If LAT would have an additional order of magnitude better sensitivity, without significant improvement in angular resolution, a situation similar to the GRB case would appear, i.e., a flat distribution of unidentified sources with a few privileged individuals only which are extensively studied in multifrequency studies. Essentially, we would find a $\gamma$-ray source coinciding with the position of every member of any population under consideration. And thus, we would lack the capability to achieve discoveries by correlation analysis. This is, perhaps, already indicating that a next generation high energy $\gamma$-ray mission after GLAST-LAT might not be exclusively sensitivitydriven if no significant improvement in angular resolution can be achieved.

The potential of this methodological procedure is not limited to the anticipated cases explicitly discussed here. By applying the proposed scheme, one can also check spurious classifications in an objective way, and test sub- 
samples among the expected classes of sources (e.g., FSRQs in correspondence of their peak radio flux, or BL Lacs in correspondence of their peak synchrotron energy, i.e. LBLs vs. HBLs, galaxy clusters in correspondence of their X-ray brightness). Summarizing, the portrayed identification scheme is not exclusively elaborated for source populations in high-energy $\gamma$-rays. It's a methodological approach to be generally applicable if the identification of source populations among a complex astrophysical dataset can only be achieved by a statistically sound discrimination between candidate classes.

\section{Acknowledgments}

DFT has been supported by Ministerio de Educación y Ciencia (Spain) under grant AYA-2006-00530, as well as by the Guggenheim Foundation.

\section{References}

1. Reimer O., Unidentified $\gamma$-ray Sources in "The Universe in Gamma Rays", Ed. V. Schönfelder, Springer A\&A Library 2001, p.319-338 ISBN 3-540-67874-3

2. Torres D. F., Romero G. E., Dame T. M., Combi J. A. \& Butt Y. M. 2003, Physics Reports 382, 303

3. Torres D. F., Reimer O., Domingo-Santamaría E. \& Digel S. 2004, ApJ 607, L99

4. Reimer O., Pohl M., Sreekumar P. \& Mattox, J. R. 2003, ApJ 588, 155

5. Torres, D.F. \& Reimer, O., 2005, ApJL 629, L141

6. Evans N.W., Ferrer F. \& Sarkar S. 2003, Physical Review D 67,103005

7. Torres D. F., Reucroft S., Reimer O. \& Anchordoqui L. A. 2003, ApJ 595, L13

8. Clay R.W. for the Pierre Auger Collaboration, Proceedings of 28th International Cosmic Ray Conference, Tsukuba 2003, p.421-424

9. Reimer, O., Proc. of High Energy Gamma-Ray Astronomy: 2nd International Symposium, Ed. F. A. Aharonian, H. J. Völk, and D. Horns. AIP Conference Proceedings, Volume 745, 2005., p.184-198

10. Feldman G. J. \& Cousins R. D. 1998, Physical Review D 57,3873

11. Gehrels N. 1986, ApJ 303, 336 